\title{
Nuevos registros de bracónidos (Hymenoptera: Braconidae) parasitoides de gusano cogollero, Spodoptera frugiperda (Lepidoptera: Noctuidae) en Durango, México
}

\author{
New records of parasitoid braconids (Hymenoptera: Braconidae) parasitoids of the fall \\ armyworm, Spodoptera frugiperda (Lepidoptera: Noctuidae) in Durango, Mexico \\ MARÍA BERENICE GONZÁLEZ-MALDONADO ${ }^{1}$; \\ JUANA MARÍA CORONADO-BLANCO ${ }^{2}$; J. REFUGIO LOMELÍ-FLORES ${ }^{3}$
}

\begin{abstract}
${ }^{1}$ Ph. D. Instituto Politécnico Nacional, CIIDIR-IPN Unidad Durango, Durango, México, mbgonzalez@ipn.mx, https://orcid.org/0000-0001-6018-0478. ${ }^{2}$ Ph. D. Universidad Autónoma de Tamaulipas, Facultad de Ingeniería y Ciencias, Centro Universitario Adolfo López Mateos, Cd. Victoria, Tamaulipas, México, jmcoronado@docentes.uat.edu.mx, https://orcid.org/0000-0002-8387-7734. ${ }^{3} \mathrm{Ph}$. D. Colegio de Posgraduados, Carr. México-Texcoco, Montecillo, Texcoco, Edo. de México, México,jrlomelif@hotmail.com, https://orcid.org/0000-0002-5888-4625.
\end{abstract}

\begin{abstract}
Autor para correspondencia
Juana María Coronado-Blanco. Ph. D. Universidad Autónoma de Tamaulipas, Facultad de Ingeniería y Ciencias, Centro Universitario Adolfo López Mateos, C. P. 87149, Cd. Victoria, Tamaulipas, México, jmcoronado@docentes uat.edu.mx, https://orcid.org/0000-0002-83877734.
\end{abstract}

\section{Citación sugerida}

GONZÁLEZ-MALDONADO, M. B.; CORONADO-BLANCO, J. M.; LOMELÍ-FLORES, J. R. 2020. Nuevos registros de bracónidos (Hymenoptera: Braconidae) parasitoides de gusano cogollero, Spodoptera frugiperda (Lepidoptera: Noctuidae) en Durango, México. Revista Colombiana de Entomología 46 (2): e8435. https://doi.org/10.25100/socolen.v46i2.8435

Recibido: 21-ago-2019

Aceptado: 19-jun-2020

Publicado: 31-dic-2020

Revista Colombiana de Entomología ISSN (Print): 0120-0488

ISSN (On Line): 2665-4385

https://revistacolombianaentomologia.univalle.edu.co

Open access

(c) (i) () BY-NC-SA 4.0

Publishers: Sociedad Colombiana de Entomología SOCOLEN (Bogotá, D. C., Colombia) https://www.socolen.org.co

Universidad del Valle (Cali, Colombia)

https://www.univalle.edu.co

(C) 2020 Sociedad Colombiana de Entomología - SOCOLEN y Universidad del Valle - Univalle
Resumen: Se relacionan las especies de la familia Braconidae (Hymenoptera: Ichneumonoidea) presentes en cultivos de maíz en el Estado de Durango, México. Se resalta la presencia de Meteorus arizonensis y Meteorus laphygmae (Euphorinae) como nuevos registros de parasitoides del gusano cogollero, Spodoptera frugiperda. Estos nuevos registros contribuyen al conocimiento de la diversidad de himenópteros, los cuales, además pueden ser utilizados en programas de control biológico.

Palabras clave: Plagas, maíz, control biológico, Lepidoptera, Hymenoptera, Braconidae, Meteorus arizonensis, Meteorus laphygmae.

Abstract: In this paper, are listed some species of the family Braconidae (Hymenoptera: Ichneumonoidea) present in corn crops in the State of Durango, Mexico. The presence of Meteorus arizonensis and Meteorus laphygmae (Euphorinae) is highlighted as new records of parasitoids of the fall armyworm, Spodoptera frugiperda. These new records contribute to the knowledge of the diversity of hymenopteran, which can also be used in biological control programs.

Keywords: Pests, corn, biological control, Lepidoptera, Hymenoptera, Braconidae, Meteorus arizonensis, Meteorus laphygmae.

\section{Introducción}

Los parasitoides Braconidae, después de Ichneumonidae, representan una de las familias de mayor riqueza taxonómica de Hymenoptera (Coronado Blanco y Zaldívar-Riverón 2014). A nivel mundial se reconocen 45 subfamilias, 1.103 géneros y 21.221 especies válidas (Yu et al. 2016). En México aunque se han realizado en los últimos años intentos importantes de clasificar y describir especies de esta familia, se encuentran sólo 36 subfamilias, 319 géneros, 707 especies determinadas y 845 morfoespecies (Coronado Blanco 2013).

Dentro de esta familia se encuentran especies del género Meteorus Haliday, 1835, parasitoides koinobiontes de coleópteros y lepidópteros, con 356 especies descritas (Aguirre et al. 2015; Yu et al. 2016). Para la región Neártica se han reportado 37 especies de Meteorus, tres de ellas como parasitoides de gusano cogollero (GutiérrezRamírez et al. 2015a; Yu et al. 2016). Sin embargo, en Durango, México hasta el momento se han registrado únicamente dos morfoespecies de Meteorus (García-Gutiérrez et al. 2013) y la especie Meteorus laphygmae Viereck, 1913 (Bahena 2008).

La complejidad en la identificación de las especies de este género se debe principalmente a los caracteres que comparten individuos de distinta especie o las diferencias entre individuos de la misma especie (polimorfismos o patrones de color). En Durango, hasta el momento sólo se ha realizado la identificación parcial de parasitoides del género Meteorus que tienen como hospedero al gusano cogollero, por lo que el objetivo de este trabajo fue realizar un estudio más detallado para identificar las especies de bracónidos parasitoides del gusano cogollero en maíz en el Estado. 


\section{Materiales y métodos}

Material biológico. El estudio se realizó en la región de los Valles, Los Llanos y las Quebradas del Estado de Durango a altitudes entre 1.750 y 2.316 msnm (seis municipios: Guadalupe Victoria, Pánuco de Coronado, Santiago Papasquiaro, Mezquital, Canatlán y Durango; y siete localidades: Morcillo, Col. Minerva, Francisco I. Madero, J. Guadalupe Aguilera, Col. Hidalgo, Carr. Mazatlán y La Ferrería, Durango), ubicados entre la latitud-longitud $24^{\circ} 57^{\prime} 46^{\prime \prime} \mathrm{N}, 105^{\circ} 25^{\prime} 8^{\prime \prime} \mathrm{O}$ y $23^{\circ} 57^{\prime} 32^{\prime \prime} \mathrm{N}, 104^{\circ} 48^{\prime} 14^{\prime \prime} \mathrm{O}$. Las recolectas de larvas de los primeros estadios de gusano cogollero se realizaron cada año en los ciclos primavera-verano 2014 a 2017, en cultivos de maíz, donde no se aplicaron insecticidas químicos, las larvas recolectadas fueron individualizadas en vasos de plástico con dieta artificial (Ashby 1972) y mantenidas en una cámara de cría (25 a $27{ }^{\circ} \mathrm{C}, 60 \%$ de H. R., fotoperiodo 14:10 O: L) en el CIIDIR-IPN Unidad Durango, hasta que alcanzaron su desarrollo completo o emergió el parasitoide. El material se encuentra depositado en el Museo de Insectos de la Facultad de Agronomía [MIFA], actualmente Facultad de Ingeniería y Ciencias de la Universidad Autónoma de Tamaulipas.

Identificación taxonómica. Se usaron las claves de Wharton et al. (1999) y la clasificación taxonómica según Yu et al. (2016). La identificación de especies de Meteorus fue corroborada a través de la comparación del material preservado con especímenes de referencia de la colección de insectos del Museo MIFA de la Universidad Autónoma de Tamaulipas y del Colegio de Posgraduados, además se utilizaron las diagnosis publicadas en Aguirre et al. (2011, 2015) y Muesebeck (1923).

\section{Resultados y discusión}

Identificación morfológica. Se recolectaron un total de 7.867 larvas, de las cuales emergieron 929 parasitoides: 669 himenópteros y 260 dípteros, de ellos se analizaron 56 especímenes $(29 / 2014,12 / 2015,2 / 2016$ y 13/2017), cuatro de la subfamilia Homolobinae (resultados no mostrados) y 52 de Euphorinae, de los cuales 31 presentaron todos los caracteres taxonómicos de Meteorus arizonensis Muesebeck, 1923 y 21 parasitoides coincidieron con la diagnosis de M. laphygmae. A continuación se presenta su distribución a nivel mundial y en México, así como algunos aspectos de su biología.

\section{Meteorus arizonensis Muesebeck, 1923}

Distribución mundial. Canadá (Alberta), Estados Unidos (Arizona, Nuevo México, Texas), México, Colombia, Honduras y Nicaragua. Región Neártica y Neotropical (Yu et al. 2016).

Distribución en México. Chihuahua (Ordoñez et al. 2015 ex S. frugiperda), Nayarit (Gutiérrez-Ramírez et al. 2015a; 2015 b - ex $S$. frugiperda) y Durango (nuevo registro - ex $S$. frugiperda).

Distribución en Durango. Canatlán, Col. Minerva, Francisco I. Madero (Pánuco de Coronado), Guadalupe Victoria, La Ferrería (Sebastián Lerdo de Tejada), Morcillo y Santiago Papasquiaro (nuevos registros). Además, nuevo registro en el Estado como parasitoide del gusano cogollero.

Comentarios. El mayor número de parasitoides se obtuvo en la Col. Minerva y Santiago Papasquiaro (ocho en cada uno). Fue la especie que se presentó en mayor abundancia (31 parasitoides).

Se observaron caracteres distintos entre especímenes de la misma especie: coloración del cuerpo, patrones de coloración en el mesosoma y tamaño del cuerpo. Es posible que debido a estos cambios de coloración anteriormente se haya identificado erróneamente dentro de M. laphygmae (Fig. 1).

Biología. Se ha reportado como parasitoide solitario de Helicoverpa zea Boddie, 1850 (Lepidoptera: Noctuidae) y $S$. frugiperda (Yu et al. 2016). a

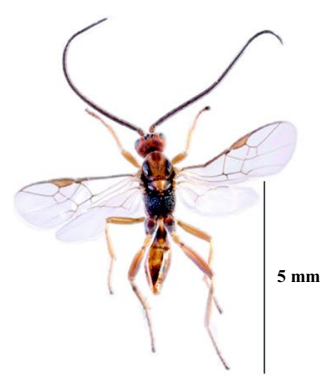

a

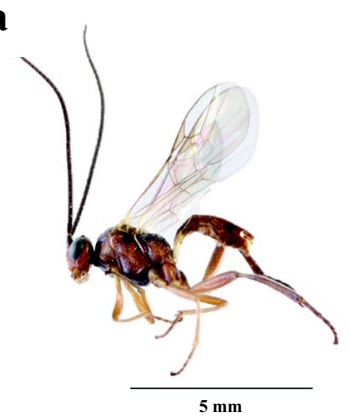

b

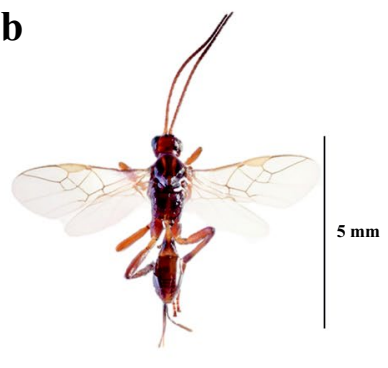

b

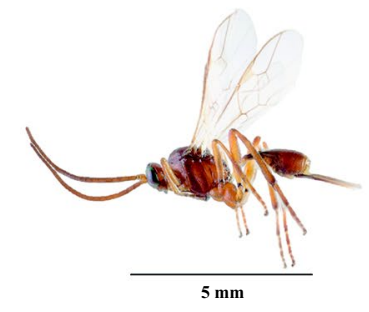

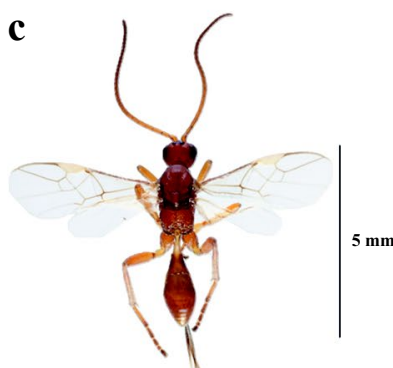

c

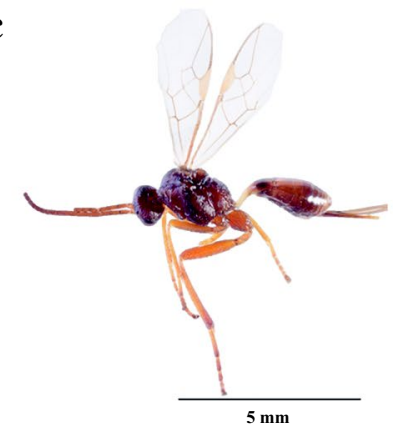

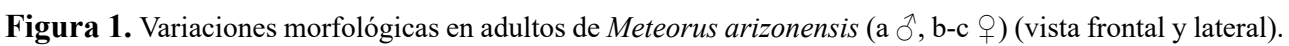


En México, han sido reportadas ocho especies de Meteorus: [M. arizonensis, M. autographae Muesebeck, 1923, M. euschausiae Muesebeck, 1923, M. hyphantriae Riley, 1887, M. kraussi Muesebeck, 1958, M. laphygmae, M. rubens (Nees, 1811) y M. versicolor (Wesmael, 1835)], de las cuales: $M$. arizonensis, $M$. autographae, $M$. laphygmae y $M$. rubens son parasitoides de gusano cogollero (Yu et al. 2016).

Las temperaturas que favorecen una alta incidencia de parasitoides de este género se encuentran entre 29 a $31^{\circ} \mathrm{C}$ en julio y agosto. Los cultivos que se encuentran en el área de Las Quebradas, en el municipio de Santiago Papasquiaro están rodeados por cerros y ríos, entre la latitud-longitud $25^{\circ} 2$ ' $34^{\prime \prime} \mathrm{N}$, $105^{\circ} 25^{\prime} 8^{\prime}$ 'O, altitud $1.750 \mathrm{msnm}$. Las temporadas de lluvias favorecieron la presencia de parasitoides, del 16 de junio al 28 de septiembre (Weatherspark 2020).

Por otro lado, en Canatlán, donde se presentó el menor número de parasitoides, la temperatura y la precipitación fueron menores a las zonas con mayor abundancia de los mismos. Canatlán tiene una altitud de $1.970 \mathrm{msnm}$ (Weatherspark 2020) y los cultivos aledaños al maíz se encuentran rodeados por frutales como manzana, membrillo y durazno, lo que podría favorecer su presencia, porque los parasitoides se alimentan de néctar. En este y los demás municipios las recolectas de larvas de gusano cogollero se realizaron en monocultivos extensivos (solo maíz).

Las claves taxonómicas utilizadas de manera adecuada permiten determinar estas especies, sin embargo las claves diseñadas hasta el momento no diferencian los cambios en coloración y patrones distintivos en el mesosoma, dichos cambios si son tomados en cuenta para diferenciar entre especies podrían confundir los resultados, por ello la importancia de tomar en cuenta otros caracteres distintivos como: venación de las alas, número de segmentos antenales, separación de los ocelos, entre otros, además de contar con investigadores expertos y comparar las especies con otros individuos en colecciones de referencia en el país y/o extranjero (GonzálezMaldonado et al. 2019).

Material examinado. $31(13 \hat{\jmath}, 18+)$, MÉXICO. Durango. Canatlán, 1.946 msnm, 9/vii/15 (19), 26/viii/17 (1ㅇ); Col. Minerva, $1.881 \mathrm{msnm}, 13 / \mathrm{vi} / 14$ (1 9 ), 12/vii/14 (2今),

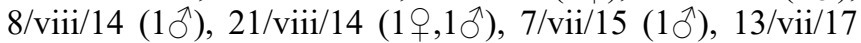
(1); Francisco I. Madero, Pánuco de Coronado, 1.891 msnm, 12/vii/14 (1ㅇ), 30/vi/15 (1ठ), 16/vi/17 (2ᄋ, $\left.1 \jmath^{\Uparrow}\right)$, Guadalupe Victoria, $2.018 \mathrm{msnm}, 9 / \mathrm{vii} / 14$ (1ठ), 30/vi/15 $\left(1 ㅇ{ }^{\Uparrow}\right)$, Morcillo, 1.925 msnm, 31/viii/16 (1ठ); La Ferrería, Sebastián Lerdo de Tejada, 1.894 msnm, 8/viii/14 (1q, 2ふ̋), 7/vii/15 (1ㅇ); Santiago Papasquiaro, $1.840 \mathrm{msnm}, 12 / \mathrm{viii} / 14$ $(1$ 잉ㄱ), 26/viii/14 (1ㅇ), 9/vii/15 (1ㅇ), 13/vii/17 (4ㅇ) , todos colectados de maíz, coll. M.B. González-Maldonado [MIFA-UAT].

\section{Meteorus laphygmae Viereck, 1913}

Distribución mundial. Estados Unidos (Louisiana, Nuevo México, Tennessee, Texas; introducida a Florida y Hawái), México, Costa Rica, Nicaragua, Honduras, Surinam, Brasil, Chile, Colombia, Venezuela. Región Neártica, Neotropical y Oceánica (Yu et al. 2016).

Distribución en México. Baja California, Chiapas, Chihuahua, Ciudad de México, Coahuila, Colima (ex S. frugiperda), Durango (ex S. frugiperda), Estado de México, Guanajuato,
Hidalgo, Jalisco, Michoacán (ex S. frugiperda), Morelos, Nayarit (ex S. frugiperda), Nuevo León, Oaxaca, Puebla, Sinaloa [ex S. exigua (Hübner)]; Sonora, Puebla, Tamaulipas (ex S. frugiperda), Veracruz, Yucatán (Alvarado-Rodríguez 1987; Carrillo 1993; Molina-Ochoa et al. 2001; 2003; Coronado Blanco et al. 2004; Hoballah et al. 2004; Bahena 2008; Jourdie et al. 2008; González et al. 2011; Rodríguez et al. 2014; Gutiérrez-Ramírez et al. 2015b; Villegas-Mendoza et al. 2015).

Distribución en Durango. Registrado por Bahena (2008) sin localidad exacta. Se registra para Col. Minerva, Francisco I. Madero (Pánuco de Coronado), Guadalupe Victoria, La Ferrería (Sebastián Lerdo de Tejada) y Santiago Papasquiaro (nuevos registros de localidades) (Fig. 2).

Comentarios. El mayor número de parasitoides se recolectó en la Col. Minerva y Santiago Papasquiaro (siete en cada uno). $M$. laphygmae tuvo un desarrollo similar a M. arizonensis, pero sus características morfológicas fueron diferentes, la más representativa fue el color amarillo en todo el cuerpo del espécimen adulto y que no existieron variaciones en los patrones de color tan marcados en el mesosoma como en $M$. arizonensis.

Biología. Por lo general, las pupas de los parasitoides emergieron en aproximadamente una semana a partir de larvas de los primeros estadios de desarrollo del gusano cogollero, $S$. frugiperda. Bahena (2008) menciona que la hembra parasita larvas pequeñas de este lepidóptero. Una vez que cumple su desarrollo y ha matado a su hospedero, emerge formando un capullo separado del cadáver; los adultos tienen hábitos nocturnos y son localizados en los hábitats de las larvas de sus hospederos lepidópteros.

Material examinado. $21 \%$, MÉXICO. Durango, Colonia Minerva, $1.881 \mathrm{msnm}, 21 / \mathrm{viii} / 14$ (3), 23/vi/15 (2+), 3/ viii/15 (1), 22/viii/16 (1q); Francisco I. Madero, Pánuco de Coronado, $1.891 \mathrm{msnm}, 7 / \mathrm{viii} / 14$ (3ㅇ), 30/vi/15 (1우); Guadalupe Victoria, $2.018 \mathrm{msnm}, 9 / \mathrm{vii} / 14$ (1), 30/vi/15 (1); La Ferrería, Sebastián Lerdo de Tejada, $1.894 \mathrm{msnm}, 8 /$ viii/14 (1 9 ); Santiago Papasquiaro, 1.840 msnm, 12/viii/14 (2q), 26/ viii/14 (5 + ), todos colectados de maíz, coll. M.B. GonzálezMaldonado [MIFA-UAT].

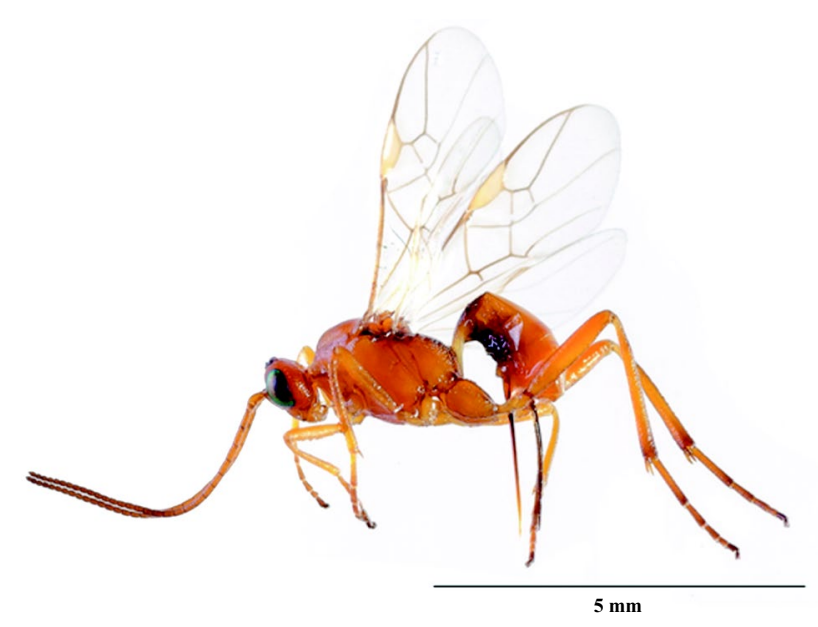

Figura 2. Adulto de Meteorus laphygmae $q$ (vista lateral). 


\section{Conclusiones}

Santiago Papasquiaro es el municipio donde se tiene la mayor abundancia de parasitoides de la familia Braconidae, específicamente del género Meteorus, reportados en este estudio. $M$. arizonensis es nuevo registro en Durango y como parasitoide del gusano cogollero en maíz, siendo una contribución al conocimiento del género Meteorus en México. Las variaciones de color en $M$. arizonensis pueden deberse a cambios en las condiciones ambientales que prevalecen en los sitios donde se desarrollan los parasitoides, tratándose de la misma especie, no siendo un carácter determinante en su identificación. La delimitación de especies de parasitoides utilizando herramientas taxonómicas combinadas con el uso de una caracterización genética permite esclarecer hipótesis y dudas taxonómicas.

\section{Literatura citada}

AGUIRRE, H.; SARMIENTO, C. E.; SHAW, S. R. 2011. Taxonomic revision and morphometric analysis of Meteorus Haliday, 1835 (Hymenoptera: Braconidae: Meteorinae) from Colombia. Zootaxa 2938 (1): 1-68. https://doi.org/10.11646/zootaxa.2938.1.1

AGUIRRE, H.; ALMEIDA, L. F. V.; SHAW, S. R.; SARMIENTO, C. E. 2015. An illustrated key to neotropical species of the genus Meteorus Haliday (Hymenoptera, Braconidae, Euphorinae). ZooKeys 489: 33-94. https://doi.org/10.3897/zookeys.489.9258

ALVARADO-RODRÍGUEZ, B. 1987. Parasites and disease associated with larvae of beet armyworm Spodoptera exigua (Lepidoptera: Noctuidae), infesting processing tomatoes in Sinaloa, México. Florida Entomologist 70 (4): 444-449. https://doi. org/10.2307/3494786

ASHBY, G. 1972. The UFAW handbook on the care and management of laboratory animals. pp. 582-587. En: Worden, A. N.; Lane-Petter, W. (Eds.). The Universities Federation for Animal Welfare, Londres.

BAHENA, J. F. 2008. Enemigos naturales de las plagas agrícolas del maíz y otros cultivos. INIFAP. Libro Técnico No. 5. Uruapan, Michoacán. 174 p.

CARRILlO, S. J. L. 1993. Síntesis del control biológico de $\mathrm{He}$ liothis spp. y Spodoptera frugiperda (J. E. Smith) (Lepidoptera: Noctuidae) en México. Folia Entomológica Mexicana (87): 85-93.

CORONADO BLANCO, J.; RUÍZ, C. E.; VARELA, F. S. 2004. Adenda a Braconidae (Hymenoptera). pp. 713-720. En: Llorente, J.; Morrone, J. J.; Yáñez, O.; Vargas, I. (Eds.). Biodiversidad, taxonomía y biogeografía de artrópodos de México: hacia una síntesis de su conocimiento. Vol. IV. UNAM. México.

CORONADO BLANCO, J. 2013. La familia Braconidae (Hymenoptera) en México. Entomología Mexicana 12 (1): 31-44.

CORONADO BLANCO, J. M.; ZALDÍVAR-RIVERÓN, A. 2014. Biodiversidad de Braconidae (Hymenoptera: Ichneumonoidea). Revista Mexicana de Biodiversidad 85: 372-378. https://doi. org/10.7550/rmb.32000

GARCÍA-GUTIÉRREZ, C.; GONZÁLEZ-MALDONADO, M. B.; GONZÁLEZ-HERNÁNDEZ, A. 2013. Parasitismo natural de Braconidae e Ichneumonidae (Hymenoptera) sobre Spodoptera frugiperda (Lepidoptera: Noctuidae). Revista Colombiana de Entomología 39 (2): 211-215. http://www.scielo.org.co/pdf/ rcen/v39n2/v39n2a06.pdf

GONZÁlEZ, H. A.; LOMELÍ, F. J.; RUÍZ, C. E. 2011. Avispas Ichneumonoidea (Insecta: Hymenoptera). pp. 441-448. En: CONABIO. La biodiversidad en Veracruz. Estudio de estado. Vol. II. CONABIO. Instituto de Ecología, A.C. México.

GONZÁLEZ-MALDONADO, M. B.; CORREA-RAMÍREZ, M. M.; ROSAS-GARCÍA. N. M.; CHAÍREZ-HERNÁNDEZ, I.; GARZÓN-ZUÑIGA, M. A. 2019. Genetic variability of species of the genus Meteorus Haliday, 1935, at Durango,
Mexico. Southwestern Entomologist 44 (4): 909-918. https:// doi.org/10.3958/059.044.0406

GUTIÉRREZ-RAMÍREZ, A.; ROBLES-BERMÚDEZ, A.; CAMBERO-CAMPOS, J.; CORONADO-BLANCO, J. M. 2015a. Meteorus arizonensis Muesebeck, 1923 (Hymenoptera: Braconidae): Nuevo registro para México. Acta Zoológica Mexicana (n.s.) 31 (1): 123-124. https://doi.org/10.21829/ azm.2015.311525

GUTIÉRREZ-RAMÍREZ, A.; ROBLES-BERMÚDEZ, A.; CAMBERO-CAMPOS, J.; SANTILLÁN-ORTEGA, C.; ORTÍZ-CATÓN, M.; CORONADO-BLANCO, J.; CAMPOS-FIGUEROA, M. 2015b. Parasitoids of Spodoptera frugiperda (Lepidoptera: Noctuidae) found in Nayarit, Mexico. Southwestern Entomologist 40 (3): 555-564. https://doi.org/10.3958/059.040.0314

HOBALLAH, M. E.; DEGEN, T.; BERGVINSON, D.; SAVIDAN, A.; TAMO, C.; TURLINGS, T. C. J. 2004. Occurrence and direct control potential of parasitoids and predators of the fall armyworm (Lepidoptera: Noctuidae) on maize in the subtropical lowlands of Mexico. Agricultural and Forest Entomology 6 (1): 83-88. https://doi.org/10.1111/j.1461-9555.2004.00207.x

JOURDIE, V.; ÁlVAREZ, N.; TURLING, T. C. J. 2008. Identification of seven species of hymenopteran parasitoids of Spodoptera frugiperda, using polymerase chain reaction amplification and restriction enzyme digestion. Agricultural and Forest Entomology 10 (2): 129-136. https://doi.org/10.1111/j.14619563.2008.00362.x

MOLINA-OCHOA, J.; HAMM, J. J.; LEZAMA-GUTIÉRREZ, R.; LÓPEZ-EDWARDS, M.; GONZÁLEZ-RAMÍREZ, M.; PESCADOR-RUBIO, A. 2001. A survey of fall armyworm (Lepidoptera: Noctuidae) parasitoids in the Mexican states of Michoacan, Colima, Jalisco, and Tamaulipas. Florida Entomologist 84 (1): 31-36. https://doi.org/10.2307/3496659

MOLINA-OCHOA, J.; CARPENTER, J. E.; HEINRICHS, E. A.; FOSTER, J. E. 2003. Parasitoids and parasites of Spodoptera frugiperda (Lepidoptera: Noctuidae) in the Americas and Caribbean basin: an inventory. Florida Entomologist 86 (3): 254289. https://doi.org/10.1653/0015-4040(2003)086[0254:PAPOS $\mathrm{F}] 2.0 . \mathrm{CO} ; 2$

MUESEBECK, C. F. W. 1923. A revision of the North American species of ichneumon-flies belonging to the genus Meteorus Haliday. Proceedings of the United States National Museum 63 (2470): 1-44. https://doi.org/10.5479/si.00963801.63-2470.1

ORDOÑEZ, G. M.; BUSTILLOS, R. J.; LOYA, M. J.; RIOS, V. C.; JACOBO, C. J. 2015. Parasitoides de Spodoptera frugiperda (J. E. Smith) (Lepidoptera: Noctuidae) en Chihuahua, México. Métodos en Ecología y Sistemática 10 (1): 67-78.

RODRÍGUEZ, M. A.; RUÍZ, C. E.; CORONADO, B. J.; TREVIÑO, C. J.; KHALAIM, A. I. 2014. Avispas Ichneumonoideas que atacan al gusano cogollero en el cultivo de maíz en México. Revista AgroProductividad 7 (1): 28-31.

VILLEGAS-MENDOZA, J. M.; SÁNCHEZ-VARELA, A.; ROSAS-GARCÍA, N. M. 2015. Caracterización de una especie de Meteorus (Hymenoptera: Braconidae) presente en larvas de Spodoptera frugiperda (Lepidoptera: Noctuidae) en el Norte de Tamaulipas, México. Southwestern Entomologist 40 (1): 161-170. https://doi.org/10.3958/059.040.0114

WHARTON, R. A.; MARSH, P. M.; SHARKEY, M. J. 1999. Manual of the new world genera of the family Braconidae (Hymenoptera). Special publication of the International Society of Hymenopterists 92 (4): 615-616. https://doi.org/10.1093/aesa/92.4.615 WEATHERSPARK. 2020. El clima promedio en Victoria de Durango. Disponible en: https://es.weatherspark.com/y/3659/Climapromedio-en-Victoria-de-Durango-M\%C3\%A9xico-durantetodo-el-a\%C3\%B1o. [Fecha revisión: 21 noviembre 2019].

YU, D. S.; ACHTERBERG, C. V.; HORSTMANN, K. 2016: Taxapad 2016, Ichneumonoidea 2015, Database on flash-drive, Ottawa, Ontario, Canada. 


\section{Origen y financiación}

El presente trabajo derivó de la línea de investigación con himenópteros que actualmente se desarrolla en el Laboratorio de Entomología del CIIDIR-IPN Unidad Durango, en el que se realizó la tesis de doctorado de María Berenice González-Maldonado (2016-2019), docente investigador del CIIDIR Durango. Este estudio fue financiado por la Universidad Autónoma de Tamaulipas, México.

\section{Contribución de los autores}

La autora María Berenice González-Maldonado fue responsable de la dirección, diseño y supervisión del trabajo experimental, revisión de resultados, procesamiento de datos e imágenes, búsqueda bibliográfica y redacción del artículo. Los autores Juana María Coronado-Blanco y J. Refugio Lomeli-Flores realizaron la identificación taxonómica del material biológico citado. 